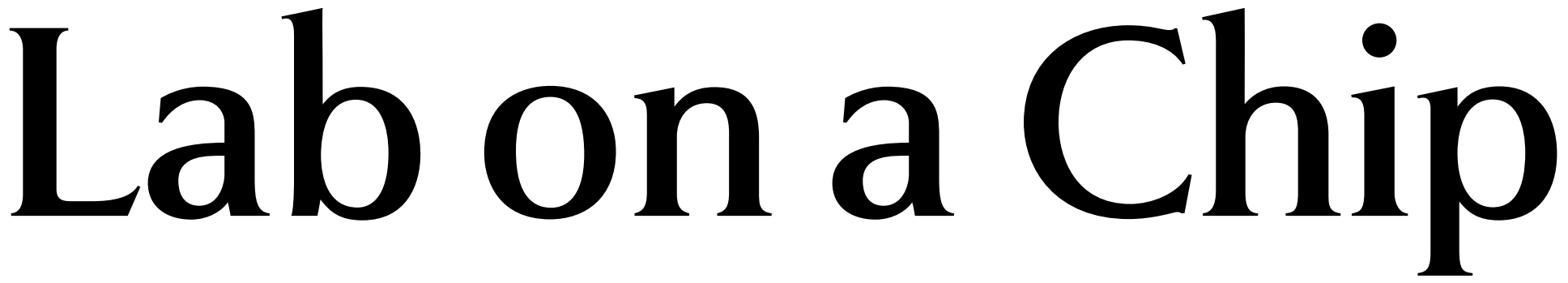

Micro- \& nano- fluidic research for chemistry, physics, biology, \& bioengineering

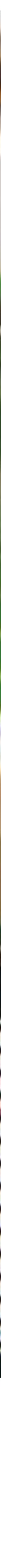




\title{
Continuous-flow electrical lysis device with integrated control by dielectrophoretic cell sorting $\dagger$
}

\author{
Guillaume Mernier, ${ }^{* a}$ Niccolò Piacentini, ${ }^{a b}$ Thomas Braschler, ${ }^{a}$ Nicolas Demierre ${ }^{a}$ and Philippe Renaud ${ }^{a}$
}

\author{
Received 15th January 2010, Accepted 28th April 2010 \\ DOI: $10.1039 / \mathrm{c000977f}$
}

\begin{abstract}
We present a device capable of electrical cell lysis and evaluation of lysis efficiency in continuous flow using dielectrophoretic cell sorting. We use a combination of AC electrical fields and so-called liquid electrodes to avoid bubble creation at the electrode surface. The electrical field distribution is calculated in different electrode configurations by numerical simulations. Cell sorting shows high lysis efficiency, $99 \%$ of yeast cells sorted after lysis featuring dielectric properties similar to dead cells.

A study of the potential device throughput is performed.
\end{abstract}

\section{Introduction}

Cell lysis has been studied for several decades, ${ }^{1,2}$ especially with regard to extracting intracellular components for analysis in fields such as metabolomics, genomics or proteomics. Various methods exist to lyse cells, such as chemical, ${ }^{3,4}$ electrical, ${ }^{5,6}$ physical $^{7,8}$ or optical ${ }^{9,10}$ lysis. Chemical lysis is easy to implement but in order to dissolve the cell membrane it makes use of reagents that can interfere with the cytosol analysis situated downstream of the lysis. Other methods such as optical lysis require more complex experimental setups.

Conversely, electrical lysis makes use of electrical fields to create pores in the cell membrane. It does not affect downstream analysis and can be implemented relatively easily, without optical components. However, this technique presents several challenges. One of the challenges is providing an electrical field high enough to affect the cell membrane while avoiding bubble formation at the electrode surface. A second challenge is the evaluation of lysis of cells featuring a cell wall around their membrane (such as yeast cells or bacteria), as no visible difference between lysed and non-lysed cells can be observed in bright field microscopy, making the assessment of lysis efficiency difficult. Various methods have been used to assess the lysis efficiency. These methods are implemented on-chip, such as fluorescence microscopy ${ }^{11}$ and impedance measurements, ${ }^{12}$ or off-chip, such as cell culture after lysis ${ }^{13}$ or detection of intracellular contents in the supernatant. ${ }^{14}$ However, none of those techniques allows on-chip evaluation of the lysis efficiency in continuous flow.

In this work we present a device able to lyse cells using AC electrical fields and so-called "liquid electrodes". Liquid electrodes are large metal electrodes patterned at the bottom of deadend chambers positioned perpendicularly to the main channel as defined by Demierre et $a .^{15}$ They provide a homogeneous

${ }^{a}$ Laboratoire de Microsystèmes LMIS4, Ecole Polytechnique Fédérale de Lausanne, Station 17, CH-1015 Lausanne, Switzerland. E-mail: guillaume.mernier@epfl.ch; Tel: +41216936581

${ }^{b}$ Dipartimento di Elettronica, Politecnico di Torino, Corso Duca degli Abruzzi 24, 10129 Torino, Italy

$\dagger$ Electronic supplementary information (ESI) available: Three images and two movies showing cell sorting without and after lysis. See DOI: $10.1039 / \mathrm{c} 000977 \mathrm{f}$ electrical field over the total channel height while keeping a simple process flow with a single planar metal layer. A cell sorting system based on dielectrophoresis situated downstream of the lysis sorts living and dead cells and thereby evaluates the percentage of yeast cells effectively lysed by the applied electrical fields. This evaluation technique is potentially very powerful to evaluate on-chip the lysis of any cell type, in continuous flow.

\section{Materials and methods}

The working solution is a Phosphate Buffer Saline (PBS) diluted in water to reach the conductivity of $55 \mathrm{mS} \mathrm{m}^{-1}$ suitable for cell sorting. The addition of $1 \%$ Bovine Albumin Serum (BSA) prevents the adhesion of cells on the microchannel walls. Yeast cells (Baker's Yeast, Saccharomyces cerevisiae) are obtained from a local grocery store. A mixed population of living and dead cells is prepared at a cell concentration of $1 \% \mathrm{w} / \mathrm{v}$. Dead yeast cells are obtained by heating a living sample for $30 \mathrm{~min}$ at $90{ }^{\circ} \mathrm{C}$. A standard protocol for exclusion assay is used to stain dead cells with trypan blue for visual identification. Propidium iodide is obtained from Sigma-Aldrich.

\section{Chip design and fabrication}

The device fabrication is described elsewhere ${ }^{16}$ and shortly repeated here. The device is made on a 4-inch glass substrate. $200 \mathrm{~nm}$-thick platinum electrodes are sputtered on top of a $20 \mathrm{~nm}$-thick Titanium adhesion layer, and further patterned by lift-off. A polydimethylsiloxane (PDMS) block covers the $20 \mu \mathrm{m}$ high microchannels made of SU8, while a plastic piece mechanically fixates the PDMS block and allows access and pressure regulation for the fluidic inlets and outlets. ${ }^{17}$ The chip design includes a lysis region, a sorting region and an observation region, as depicted in Fig. 1. Cell lysis is performed using 5 liquid electrodes on each side whereas dielectrophoretic sorting uses 10 electrodes on each side.

\section{Theoretical considerations and numerical simulations}

Cell lysis. An important point to consider is the frequency of the lysis voltage. Cell lysis is optimal in direct current (DC) fields because the field is continuously above the threshold of membrane electroporation. On the other hand, in DC a relatively 


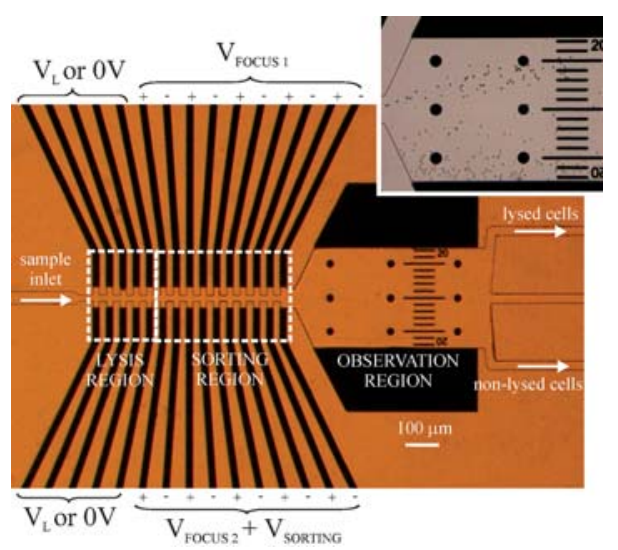

Fig. 1 Photograph of the chip design, showing the different regions through which cells pass, as well as the liquid electrodes used to apply the lysis and dielectrophoresis voltages. Inset: living and dead cells flowing in the observation region after sorting.

low voltage applied at the microelectrodes can cause bubble creation at the electrode surface. To avoid this unwanted effect, an alternating current (AC) voltage can be used but its frequency should be low enough to keep a strong effect on the cell membrane, i.e. the voltage should stay long enough above the membrane electroporation threshold to be able to charge the membrane capacitance.

The transmembrane potential (TMP) leading to membrane electroporation is in the range of $1 \mathrm{~V}^{18}$ In $\mathrm{DC}$, this potential in given by $\Delta \phi=1.5 R E_{0} \cos \theta$, where $R$ is the cell radius, $E_{0}$ the local electrical field and $\theta$ is the polar angle measured from the center of the cell with respect to the direction of the field. Pores appear first at locations where the TMP is maximum, i.e. where $\cos \theta=1$.

In $\mathrm{AC}$, the TMP is given by $\Delta \phi=1.5 R E_{0} \cos \theta /\left(1+(\omega \tau)^{2}\right)^{1 / 2},{ }^{19}$ where $\omega$ is the pulsation of the field, and $\tau$ is the time constant given by ${ }^{20} \tau=R C_{m}\left(\rho_{\text {int }}+\rho_{\text {ext }} / 2\right)$, with $C_{m}$ being the specific cell membrane capacitance, and $\rho_{\text {int }}$ and $\rho_{\text {ext }}$ the cytosol and suspension medium resistivities, respectively. In order to keep the TMP in AC as close as in the DC case, the criteria on the lysis frequency is $\omega \tau \ll 1$. The dependence of the transmembrane potential with the frequency is shown in Fig. 2A for yeast cells and an electrical field of $2 \mathrm{kV} \mathrm{cm}^{-1}$. The parameters for yeast cells found in the literature ${ }^{21}$ are summarised in Fig. 2A and give a time constant $\tau=3.3 \times 10^{-7} \mathrm{~s}$, so that the lysis frequency should be $f \ll 1 /(2 \pi \tau)=500 \mathrm{kHz}$. The lysis frequency is chosen at $50 \mathrm{kHz}$, above which significantly higher voltages have to be applied in order to achieve cell lysis.

Cell sorting. The dielectrophoretic force is used to separate living and dead cells after lysis, based on their different dielectric properties. The time average of this force in an inhomogeneous and time-varying electrical field $E$ is given by the following equation: ${ }^{22}$

$$
\left\langle F_{D E P}(t)\right\rangle=\pi \varepsilon_{m} r^{2} \operatorname{Re}\left(K_{\mathrm{CM}}\right) \nabla|E|^{2}
$$

where $\varepsilon_{m}$ is the permittivity of the medium, $r$ the radius of the particle, and $\operatorname{Re}\left(K_{C M}\right)$ is the real part of the Clausius-Mossotti factor defined as
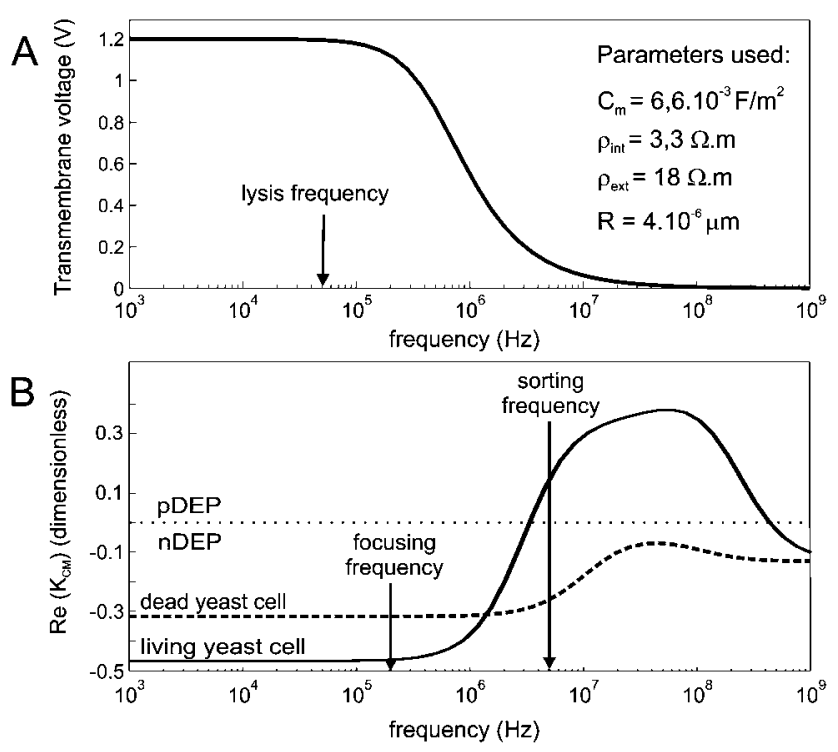

Fig. 2 A. Transmembrane potential caused by an electrical field of $2 \mathrm{kV} \mathrm{cm}{ }^{-1}$; B. Real part of the Clausius-Mossotti factor for living and dead yeast cells.

$$
K_{C M}=\frac{\varepsilon_{p}-\underline{\varepsilon}_{m}}{\underline{\varepsilon}_{p}+2 \underline{\varepsilon}_{m}}, \underline{\varepsilon}=\varepsilon-j \frac{\sigma}{\omega}
$$

$\varepsilon$ being the permittivity and $\sigma$ the conductivity of the particle and the medium; $\omega$ is the angular frequency of the electric field, $j$ the imaginary unit. $K_{C M}$ is bounded between -0.5 and 1 , and can give rise to DEP forces in two opposite directions. Namely one refers to positive dielectrophoresis (pDEP) when cells are attracted in regions where the gradient of electric field is large. Conversely, cells are repelled from those regions in case of negative dielectrophoresis ( $\mathrm{nDEP}$ ).

The dielectrophoretic sorting takes advantage of the different dielectric properties of living and dead cells. Dead cells feature a leaky membrane having much higher conductivity than living cells. The membrane is more permeable and allows out-diffusion of intracellular components, eventually leading to cell death when the cell has no more physiological activity. The cytosol of a dead cell has therefore the same characteristics as the suspension medium, in this case a lower conductivity than in living cells. Fig. 2B shows the real part of $K_{C M}$ for living and dead yeast cells as a function of the frequency, according to the multi-shell model described by Huang et al. ${ }^{23}$

In the $200 \mathrm{kHz}$ frequency range, the electrical field is blocked by the cell membrane and both living and dead yeast cells experience the same nDEP force, allowing focusing of the cell stream in an equilibrium position. In the $5 \mathrm{MHz}$ frequency range, the electrical field can pass through the membrane and the cytosol influences the dielectrophoretic force. Dead yeast cells still undergo nDEP, whereas living cells, which have a more conductive cytoplasm, are subjected to pDEP forces attracting them towards the other direction. In this work the focusing and sorting signals are applied on the electrodes as shown on Fig. 1. Voltages and frequencies are obtained experimentally to achieve best separation, as explained in more detail in a previous paper. $^{21}$ 
It is interesting to note that as the lysis voltage is applied in $\mathrm{AC}$, it also induces a dielectrophoretic effect on the cells. At the lysis frequency of $50 \mathrm{kHz}$, both cell types experience negative dielectrophoresis, as shown in Fig. 2B. This implies that the cells are repelled from places with high electrical field gradients in the lysis region.

Numerical simulations. Numerical simulations are performed to evaluate the electrical field achieved by the lysis voltage. A two-dimensional finite element model is studied with the simulation software package COMSOL Multiphysics and allows the calculation of the electrical field distribution from the applied voltages.

The electrical connections of the lysis electrodes can be implemented in two ways; a first configuration connects the electrodes alternately, so that the current is passing between the neighboring electrodes, like it is done for the electrodes for dielectrophoresis. The second way is to connect all electrodes on the same side of the channel to the same potential, so that the electrical field lines are crossing the main channel perpendicularly. This latter facing electrodes configuration has the advantage of requiring only 2 electrodes covering all the lateral channels of the liquid electrodes.

Fig. 3 and 4 show the electrical field distribution in the alternating electrodes and facing electrodes configurations and the field profile in the center of the channel, showing maximum electrical fields of $2.7 \mathrm{kV} \mathrm{cm}^{-1}$ and $1.9 \mathrm{kV} \mathrm{cm}^{-1}$, respectively, for an applied voltage of $20 \mathrm{~V}$. In the face-to-face configuration, the highest electrical field is obtained in the main channel at the level of the lateral channels of the liquid electrodes, whereas in the alternating configuration the field is higher in the main channel between the lateral channels. This influences the behavior of the cells in the lysis region, as they are repelled from high electrical field gradients by dielectrophoresis. Adapting the flow speed allows to pass cells through these regions and the dielectrophoretic effect can be used to retain the cells in the lysis region.

Experimental results and discussion. We propose a novel way to evaluate the lysis of yeast cells using sorting by multiplefrequency dielectrophoresis. This method uses the different dielectric properties of living and dead cells, and has been shown to be very efficient in separating living and dead yeast cells. ${ }^{21,24}$

If cells feature dielectric properties of dead cells (leaky membrane, cytosol similar to suspension medium) after lysis, the extraction of the intracellular components is considered successful and cells are considered to be lysed. As our interest is the effective extraction of intracellular components, no further kinetic study is performed to differentiate between temporary electroporation and irreversible cell lysis. Lysis efficiency is defined here as the percentage of cells featuring dielectric characteristics similar to dead cells after passing through the lysis region.

Dead yeast cells are stained with trypan blue and added to the sample for sorting calibration. They also act as a control to validate the similar characteristics observed in lysed cells. A mixture of living and dead cells is injected at the inlet of the device. As shown in Fig. 1, cells pass first through a lysis region and later through a sorting region. The presence of a wider observation region after the sorting allows an amplification of the difference in output position between living and dead cells, and reduces the flow speed for better observation. Living cells and dead cells are counted by optical observation under an

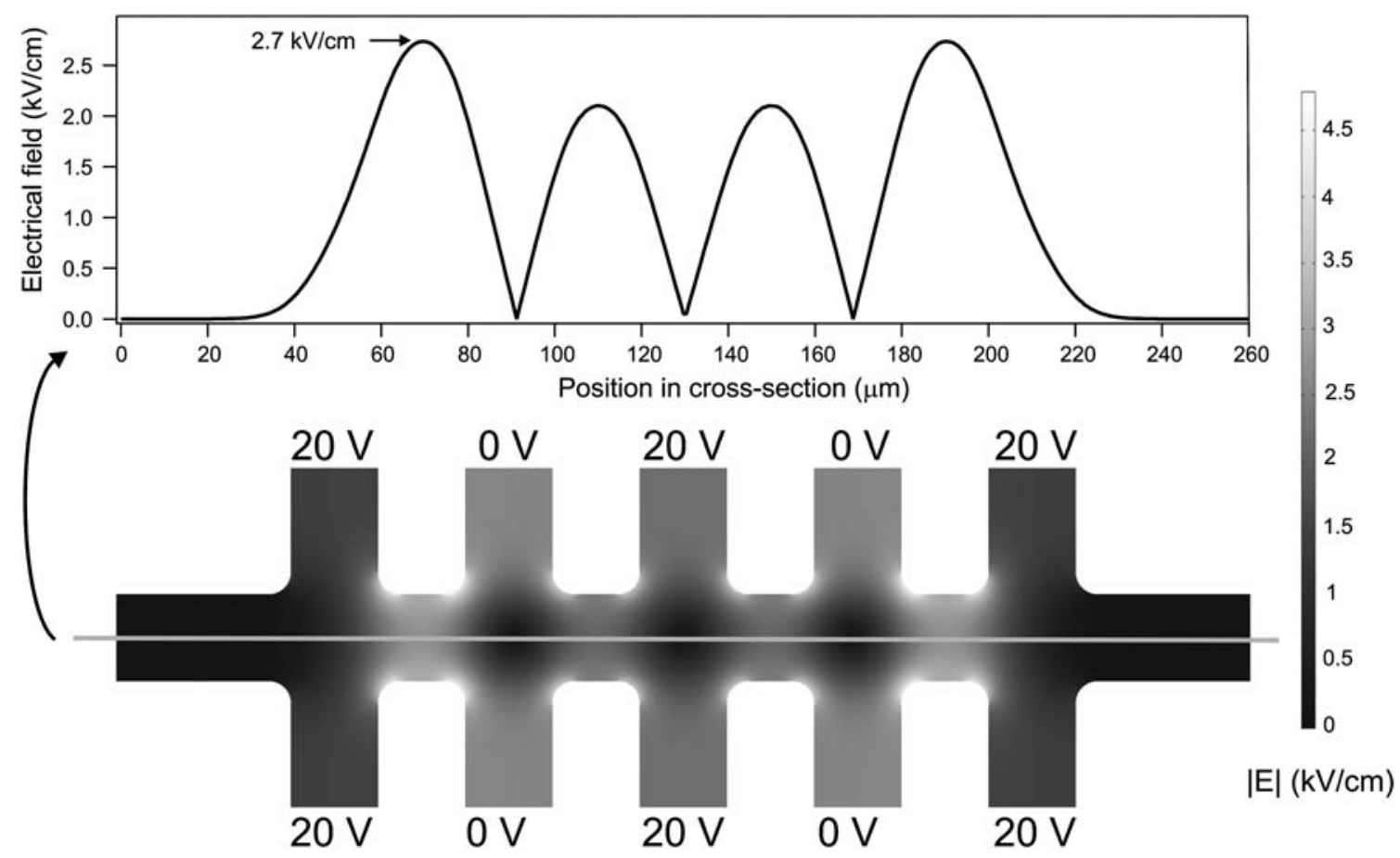

Fig. 3 Electrical field distribution in the alternating electrodes configuration, with the electrical field profile in the center of the main channel for a voltage $V_{\mathrm{L}}=20 \mathrm{~V}$ (corresponding to the maximum for an AC voltage of $40 V_{\mathrm{pp}}$ ). 


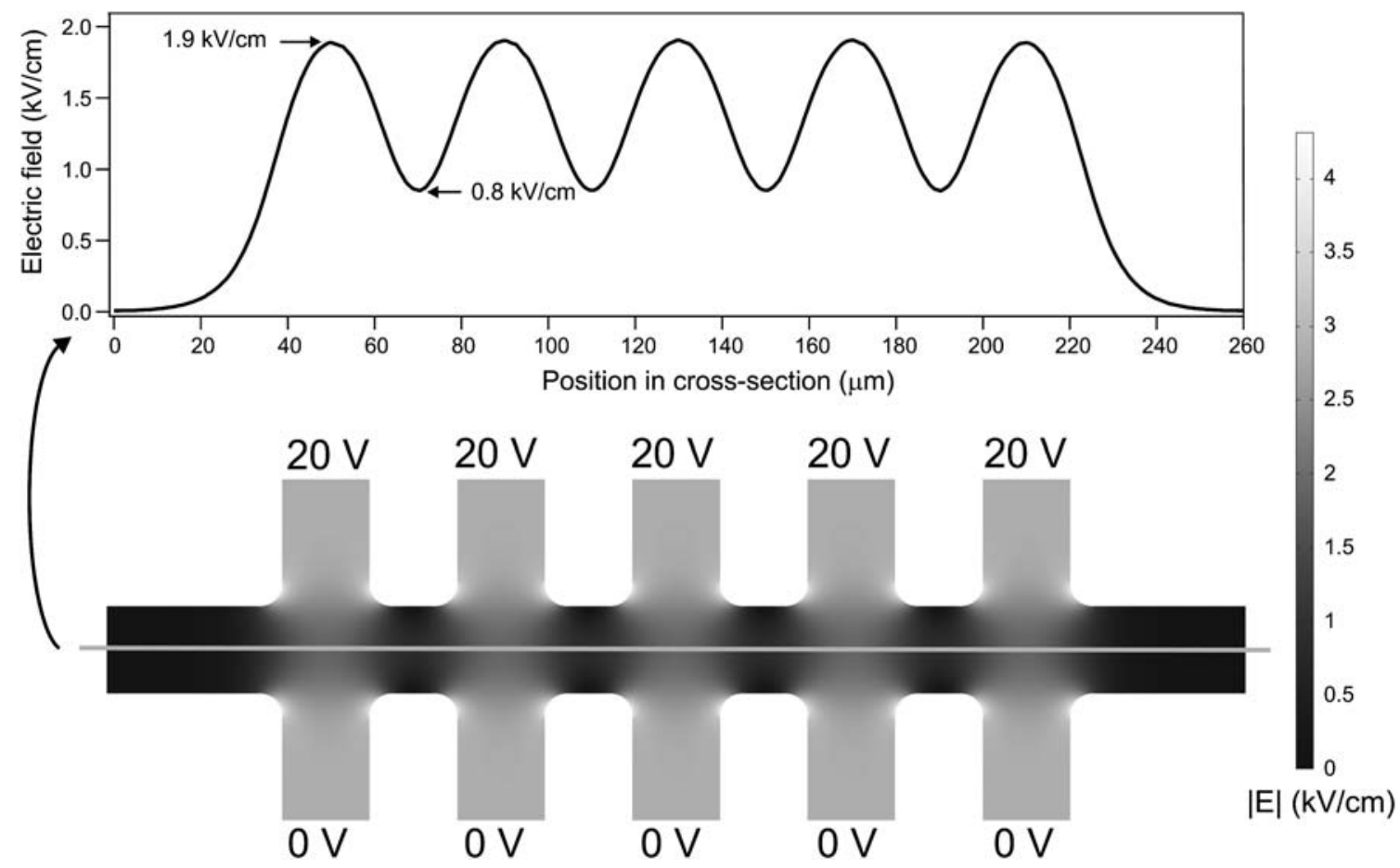

Fig. 4 Electrical field distribution in the facing electrodes configuration, with the electrical field profile in the center of the main channel for a voltage $V_{\mathrm{L}}=20 \mathrm{~V}$ (corresponding to the maximum for an AC voltage of $40 V_{\mathrm{pp}}$ ).

inverted microscope (Leica) using a uEye CCD camera (IDS Imaging), and differentiated thanks to the trypan staining performed beforehand on the injected sample. Their output positions are recorded and summarised in histograms for the different applied voltages and electrode configurations.

Fig. 5A shows the output positions after sorting when no lysis voltage is applied. The graph shows a clear difference in output position between stained and non-stained cells, characterised by two distinct peaks in the histogram, and gives a viability of $70 \%$ corresponding to the one of the injected sample. Cells experiencing a strong force on one side will flow close to the edge of the channel (as they cannot go further to the side) and the last position range is therefore a popular result.

When a high voltage ( $55 V_{\mathrm{pp}}$ ) is applied in facing (Fig. 5B) or alternating (Fig. 5C) electrodes configuration, the equilibrium position of the non-stained cells moves to the left side, indicating that they have dielectric properties similar to dead cells, although they were alive when injected into the device. If we consider that all cells on the left half are dead and all cells on the right side are alive, the percentage of living cells effectively lysed by the device can be deduced. Table 1 summarises the parameters for these three experiments. The number of non-stained cells which are effectively lysed is calculated as being $\geq 99 \%$ for both configurations. This result is validated by the injection of propidium iodide at the device outlet, showing all cells are dead after the lysis region.

The comparison with the numerical simulations provides an estimate of the maximum electrical field achieved in the center of the channel, equal to 3.7 and $2.6 \mathrm{kV} \mathrm{cm}^{-1}$ in the alternating and facing electrodes configuration, respectively. The corresponding theoretical transmembrane potentials are $2.2 \mathrm{~V}$ and $1.5 \mathrm{~V}$, respectively.

These results show very high lysis efficiency but are performed with relatively low flow speeds, around $300 \mu \mathrm{m} \mathrm{s}^{-1}$ in the lysis and sorting regions. An assessment of the lysis efficiency of this device at higher flow speed (around $5 \mathrm{~mm} \mathrm{~s}^{-1}$ ) is studied, and the output positions of cells after lysis are shown in Fig. 6. The sorting efficiency is lower because the dielectrophoretic sorting force is smaller compared to the convective force of the flow and cells have less time to find an equilibrium position, even if the sorting voltages are adapted to higher values, as shown on Table 2 .

The limit of output positions between dead and living cells is now chosen at a distance of $40 \mu \mathrm{m}$ from the centre of the channel, as it is where the control experiment features an inversion in dominant population. The lysis efficiency is here more difficult to assess as the sorting is not working perfectly anymore, but the effect of the lysis voltages is clearly visible and the lysis effect seems not to be influenced by the higher throughput.

The alternating electrodes configuration gives a more centered output position histogram (see Fig. 6C), which can be due to several reasons. On the first hand, the application of high electrical fields in the alternating electrodes configuration focuses the cells very strongly in the center of the channel, and the sorting voltages might not be strong enough to deviate the cells much from this centered position at high flow speeds. On the other hand, the lysis might be less efficient despite the higher maximum fields achieved because the minimum electrical fields are much smaller in front of the dead-end channels (as shown in Fig. 3) and may be smaller than the electroporation threshold. Another inconvenience of the alternating electrodes configuration is the 

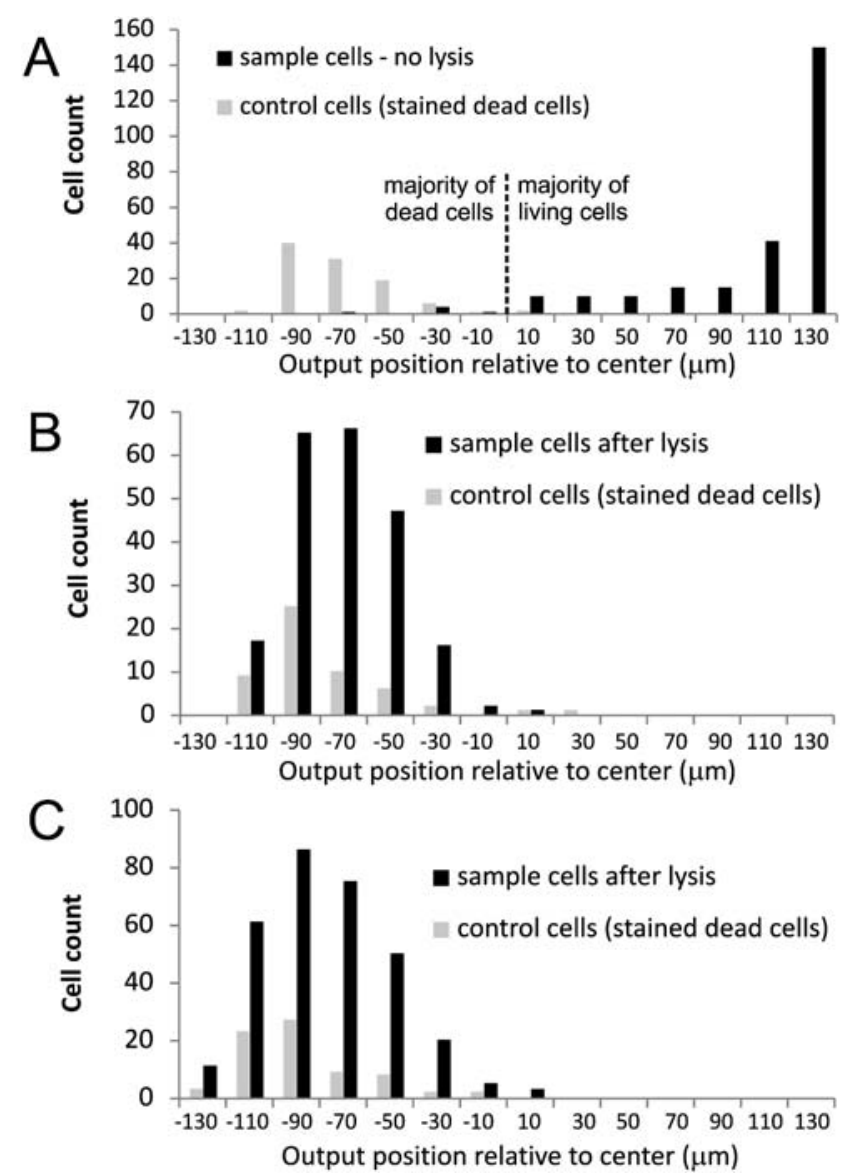

Fig. 5 Histogram showing cell counts at 14 different output positions after sorting by dielectrophoresis at flow speeds around $300 \mu \mathrm{m} \mathrm{s}^{-1}$ in the lysis and sorting region. A: no lysis voltage; B: square lysis voltage of $55 V_{\mathrm{pp}}$ at $50 \mathrm{kHz}$ using the facing electrodes configuration; C: square lysis voltage of $55 V_{\mathrm{pp}}$ at $50 \mathrm{kHz}$ using the alternating electrodes configuration.

accumulation of cells in the first lateral dead-end channel, due to the lower electrical field gradients present there (see Fig. S1 in the ESI $\dagger$ ).

The lysis region and sorting region are $200 \mu \mathrm{m}$ and $400 \mu \mathrm{m}$ long, respectively and the measured flow speed is around $5 \mathrm{~mm} \mathrm{~s}^{-1}$, giving transit times in the two regions of 40 and $80 \mathrm{~ms}$, respectively. The transit time in the lysis region is still long enough for the lysis to occur, and the transit time in the sorting region is long enough for the cytosol to diffuse out of the cells so that lysed cells feature dielectric properties similar to dead cells. The number of

Table 1 Parameters for the three experiments at low flow speeds, around $300 \mu \mathrm{m} \mathrm{s}^{-1}$

\begin{tabular}{|c|c|c|c|c|c|}
\hline $\begin{array}{l}\text { Lysis } \\
\text { voltage } V_{L}\end{array}$ & $\begin{array}{l}\text { cells } \\
\mathrm{s}^{-1}\end{array}$ & $\begin{array}{l}\% \\
\text { of lysis } \\
\text { efficiency }\end{array}$ & $\begin{array}{l}\text { Focusing } \\
\text { voltage } \\
\text { V }_{\text {FOCUS } 1}\end{array}$ & $\begin{array}{l}\text { Focusing } \\
\text { voltage } \\
\text { V }_{\text {FOcus } 2}\end{array}$ & $\begin{array}{l}\text { Sorting } \\
\text { voltage } \\
\text { V }_{\text {SORTING }}\end{array}$ \\
\hline $\begin{array}{c}\text { No lysis } \\
(0 \mathrm{~V})\end{array}$ & 6.8 & - & $\begin{array}{l}3 \mathrm{~V} @ \\
200 \mathrm{kHz}\end{array}$ & $\begin{array}{l}3.5 \mathrm{~V} @ \\
200 \mathrm{kHz}\end{array}$ & $\begin{array}{c}2.8 \mathrm{~V} @ \\
5 \mathrm{MHz}\end{array}$ \\
\hline $\begin{array}{c}55 V_{\mathrm{pp}} \\
\text { facing } \\
\text { elec. }\end{array}$ & 5.1 & 99.5 & $\begin{array}{l}3 \mathrm{~V} @ 200 \mathrm{kHz} \\
\quad\end{array}$ & $\begin{array}{l}3.5 \mathrm{~V} @ \\
200 \mathrm{kHz}\end{array}$ & $\begin{array}{l}2.8 \mathrm{~V} @ \\
5 \mathrm{MHz}\end{array}$ \\
\hline $\begin{array}{c}55 V_{\mathrm{pp}} \\
\text { altern. } \\
\text { elec. }\end{array}$ & 7.3 & 99.0 & $\begin{array}{l}3 \mathrm{~V} @ \\
\quad 200 \mathrm{kHz}\end{array}$ & $\begin{array}{l}3.5 \mathrm{~V} @ \\
200 \mathrm{kHz}\end{array}$ & $\begin{array}{l}2.8 \mathrm{~V} @ \\
5 \mathrm{MHz}\end{array}$ \\
\hline
\end{tabular}
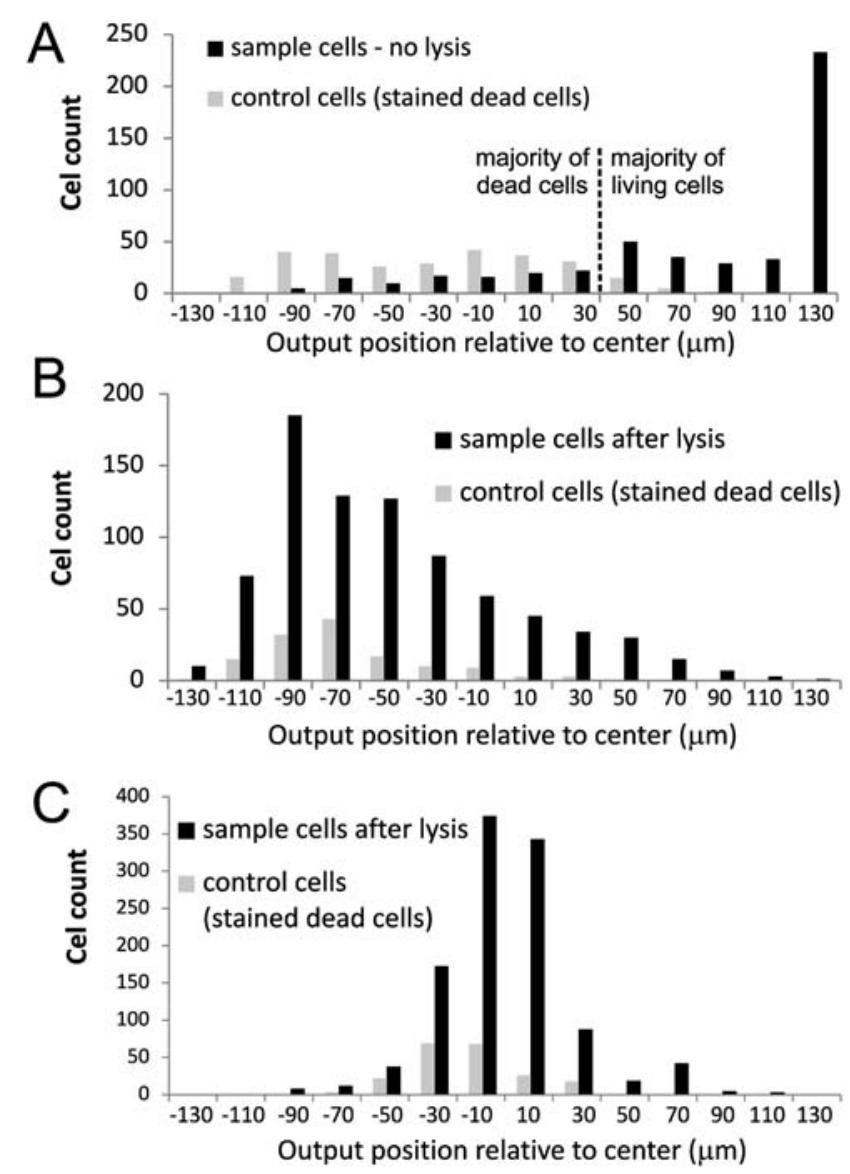

Fig. 6 Histogram showing cell counts at 14 different output positions after sorting by dielectrophoresis at flow speeds around $5 \mathrm{~mm} \mathrm{~s}^{-1}$. A: no lysis; B: square lysis voltage of $100 V_{\mathrm{pp}}$ at $50 \mathrm{kHz}$ using the facing electrodes configuration; $\mathrm{C}$ : square lysis voltage of $100 \mathrm{~V}_{\mathrm{pp}}$ at $50 \mathrm{kHz}$ using the alternating electrodes configuration; Influence of the higher throughput is clearly visible in the control experiment, where the sorting is less efficient. However the effect of the lysis voltage remains very clear.

Table 2 Parameters for the three experiments at higher flow speeds, around $5 \mathrm{~mm} \mathrm{~s}^{-1}$

\begin{tabular}{|c|c|c|c|c|c|}
\hline $\begin{array}{l}\text { Lysis } \\
\text { voltage } \\
\mathrm{V}_{\mathrm{L}} \\
\end{array}$ & $\begin{array}{l}\text { cells } \\
\mathrm{s}^{-1}\end{array}$ & $\begin{array}{l}\% \\
\text { of lysis } \\
\text { efficiency }\end{array}$ & $\begin{array}{l}\text { Focusing } \\
\text { voltage } \\
\mathrm{V}_{\text {FOCUS } 1}\end{array}$ & $\begin{array}{l}\text { Focusing } \\
\text { voltage } \\
\mathrm{V}_{\text {FOCUS 2 }}\end{array}$ & $\begin{array}{l}\text { Sorting } \\
\text { voltage } \\
\mathrm{V}_{\text {SORTING }}\end{array}$ \\
\hline $\begin{array}{l}\text { No lysis } \\
(0 \mathrm{~V})\end{array}$ & 51.8 & - & $\begin{array}{l}11 \text { V@ } \\
200 \mathrm{kHz}\end{array}$ & $\begin{array}{l}7 \mathrm{~V} @ \\
200 \mathrm{kHz}\end{array}$ & $\begin{array}{c}7.7 \mathrm{~V} @ \\
5 \mathrm{MHz}\end{array}$ \\
\hline $\begin{array}{c}100 V_{\mathrm{pp}} \\
\text { facing } \\
\text { elec. }\end{array}$ & 85.3 & 93.8 & $11 \mathrm{V@@}$ & $\begin{array}{l}7 \mathrm{~V} @ \\
\quad 200 \mathrm{kHz}\end{array}$ & $\begin{array}{c}7.7 \mathrm{~V} @ \\
5 \mathrm{MHz}\end{array}$ \\
\hline $\begin{array}{c}100 V_{\mathrm{pp}} \\
\text { altern. } \\
\text { elec. }\end{array}$ & 97.6 & 93.0 & 11 V@@ kHz & $\begin{array}{l}7 \mathrm{~V} @ \\
\quad 200 \mathrm{kHz}\end{array}$ & $\begin{array}{c}7.7 \mathrm{~V} @ \\
5 \mathrm{MHz}\end{array}$ \\
\hline
\end{tabular}

liquid electrodes dedicated to lysis and dielectrophoretic sorting can be further increased to achieve longer transit times or to work at higher throughput.

\section{Conclusions}

We have developed a device able to perform cell lysis using a novel "liquid electrodes" design. An AC electrical field has been 
used at a frequency optimising the cell lysis while avoiding the creation of bubbles at the electrode surface. This AC field causes a dielectrophoretic effect on the cells that can be used to increase the transit time of the cell in the lysis region. Yeast cell lysis has been performed and evaluated by dielectrophoretic sorting. The lysis efficiency has been shown to be very efficient for the two electrode configurations studied, even at higher throughput.

The sorting of living and dead cells provides a unique tool for the evaluation of yeast cell lysis in continuous flow. It is suitable to study effective extraction for cytosol as the sorting highlights the different dielectric properties of the cells, such as the cytosol conductivity. This evaluation method could also be used in the assessment of the lysis of other cells, such as bacteria or plant cells.

\section{Acknowledgements}

The research leading to these results has received funding from the European Community's Seventh Framework Programme (FP7/2007-2013) under grant agreement no. 227243. Thanks to the Centre of MicroNanoTechnology for access to the cleanroom facilities.

\section{References}

1 U. Zimmermann, G. Pilwat, C. Holzapfel and K. Rosenheck, Electrical Hemolysis of Human and Bovine Red Blood Cells, J. Membr. Biol., 1976, 30, 135-152.

2 M. R. J. Salton, Cell structure and the enzymic lysis of bacteria, J. Gen. Microbiol., 1953, 9(3), 512-523.

3 T. A. Bramley, R. Colemana and J. B. Fineana, Chemical, enzymological and permeability properties of human erythrocyte ghosts prepared by hypotonic lysis in media of different osmolarities, Biochim. Biophys. Acta, Biomembr., 1971, 241(3), 752-769.

4 D. Irimia, R. G. Tompkins and M. Toner, Single-cell chemical lysis in picoliter-scale closed volumes using a microfabricated device, Anal. Chem., 2004, 76(20), 6137-6143.

5 E. Jeltsch and U. Zimmermann, Particles in a Homogeneous Electrical Field: A Model for the Electrical Breakdown of Living Cells in a Coulter Counter, J. Electroanal. Chem., 1979, 104, 349-384.

6 F. T. Han, Y. Wang and C. D. Sims, Fast electrical lysis of cells for capillary electrophoresis, Anal. Chem., 2003, 75(15), 3688-3696.

7 B. A. Pethica, Lysis by physical and chemical methods, J. Gen. Microbiol., 1958, 18(2), 473-480.
8 D. Di Carlo, K. H. Jeong and L. P. Lee, Reagentless mechanical cell lysis by nanoscale barbs in microchannels for sample preparation, $L a b$ Chip, 2003, 3(4), 287-291.

9 K. R. Rau, A. Guerra and A. Vogel, Investigation of laser-induced cell lysis using time-resolved imaging, Appl. Phys. Lett., 2004, 84(15), 2940-2942.

10 M. D. Dhawan, F. Wise and A. J. Baeumner, Development of a laserinduced cell lysis system, Anal. Bioanal. Chem., 2002, 374(3), 421-426.

11 H.-Y. Wang, A. K. Bhunia and C. Lu, A microfluidic flow-through device for high throughput electrical lysis of bacterial cells based on continuous de voltage, Biosens. Bioelectron., 2006, 22, 582-588.

12 J. Suehiro, M. Shutou, T. Hatano and M. Hara, High sensitive detection of biological cells using dielectrophoretic impedance measurement method combined with electropermeabilization, Sens. Actuators, B, 2003, 96, 144-151.

13 S.-W. Lee and Y.-C. Tai, A micro cell lysis device, Sens. Actuators, A, 1999, 73, 74-79.

14 L. C. Waters, S. C. Jacobson and N. Kroutchinina, Microchip device for cell lysis, multiplex PCR amplification, and electrophoretic sizing, Anal. Chem., 1998, 70(1), 158-162.

15 N. Demierre, T. Braschler, P. Linderholm, U. Seger, H. van Lintel and P. Renaud, Characterization and optimization of liquid electrodes for lateral dielectrophoresis, Lab Chip, 2007, 7(3), $355-365$.

16 N. Demierre, T. Braschler, R. Muller and P. Renaud, Focusing and continuous separation of cells in a microfluidic device using lateral dielectrophoresis, Sens. Actuators, B, 2008, 132, 388-396.

17 T. Braschler, L. Metref, R. Zvitov-Marabi, H. van Lintel, N. Demierre, J. Theytaz and P. Renaud, A simple pneumatic setup for driving microfluidics, Lab Chip, 2007, 7, 420-422.

18 U. Zimmermann, Electrical breakdown, electropermeabilization and electrofusion, Rev. Physiol. Biochem. Pharmacol, 1986, 105, 176-256.

19 C. Holzapfel, J. Vienken and U. Zimmermann, Rotation of Cells in an Alternating Electric-Field - Theory and Experimental Proof, J. Membr. Biol., 1982, 67(1), 13-26.

20 E. Jeltsch and U. Zimmermann, Particles in a homogeneous electrical field: A model for the electrical breakdown of living cells in a Coulter counter, Bioelectrochem. Bioenerg., 1979, 6, 349-384.

21 T. Braschler, N. Demierre, E. M. Nascimento, T. Silva, A. G. Oliva and P. Renaud, Continuous separation of cells by balanced dielectrophoretic forces at multiple frequencies, Lab Chip, 2008, 8 , 280-286.

22 P. Gascoyne and J. Vykoudal, Particle separation by dielectrophoresis, Electrophoresis, 2002, 23(13), 1973-1983.

23 Y. Huang, R. Holzel, R. Pethig and X. B. Wang, Differences In The Ac Electrodynamics Of Viable And Nonviable Yeast-Cells Determined Through Combined Dielectrophoresis And Electrorotation Studies, Phys. Med. Biol., 1992, 37(7), 1499-1517.

24 G. Mernier, N. Piacentini, R. Tornay, N. Buffi and P. Renaud, Cell Viability Assessment By Flow Cytometry Using Yeast As Cell Model, Sens. Actuators, B, 2009, DOI: 10.1016/ j.snb.2009.11.066. 